\title{
Integrating Radio Positioning and Communications: New Synergies
}

\author{
Ronald Raulefs, ${ }^{1}$ Simon Plass, ${ }^{1}$ and Marco Luise ${ }^{2}$ \\ ${ }^{1}$ German Aerospace Center (DLR), Institute of Communications and Navigation, Oberpfaffenhofen, 82234 Wessling, Germany \\ ${ }^{2}$ Dipartimento di Ingegneria dell'Informazione, Università degli Studi di Pisa, 56100 Pisa, Italy
}

Correspondence should be addressed to Ronald Raulefs, ronald.raulefs@dlr.de

Received 14 November 2010; Accepted 14 November 2010

Copyright (c) 2010 Ronald Raulefs et al. This is an open access article distributed under the Creative Commons Attribution License, which permits unrestricted use, distribution, and reproduction in any medium, provided the original work is properly cited.

\section{Integrating Radio Positioning and Communications: New Synergies}

Wireless communications and positioning systems and services, although often coexisting into the same terminal and sharing to some extent signal formats, have different constraints to cope with. Communications systems usually target high spectral efficiency (in terms of data rate per occupied bandwidth) with specific requirements such as low latency, low bit error rate, and low power consumption. Positioning terminals either base their functionality on very accurate synchronization with the received signals for acquisition and tracking, or they derive location information from previous knowledge of the wireless system or even obtain their position from knowledge of the radio propagation channel. The two technologies have different goals and, therefore, achieve different solutions.

However, communications and localization have a high potential to benefit from each other and appear more and more to be intertwined. This holds for physical (PHY) and medium access control (MAC) layer issues as well as for the application layer. The convergence of communications and positioning in the PHY and MAC layers have gradually attracted research interest and represent the enabling factor for the application layer. In fact, positioning information is an emerging "must-have" for numerous applications in smart phones (location-based applications), and companies are developing their business models on the ubiquitous access of data and of position information. Mobile radio services are already using nowadays positioning information for intelligent transport systems, location sensitive billing, and location-based advertising, just to mention a few.
The papers in this special issue represent a valid snapshot of current research in the field, showing the new synergies of integrated radio positioning and wireless communications.

The papers are grouped into three clusters: (i) practical investigations in real networks to show the performance gains based on fingerprinting techniques, (ii) focusing on the coexistence of different communications systems and global navigation satellite systems (GNSS), and (iii) underlay cognitive radio system using positioning information to support the secondary user.

In the first group of papers, the studies are carried out by practical investigations focusing on trials and realisation effort.

I. Ahriz et al. study handset localization with high accuracies by using $2 \mathrm{G}$ cellular mobile radio systems. The paper focuses on an indoor environment-urban apartmentsto support health care applications. All GSM carriers are used as fingerprints. Even by reducing the numbers of GSM carriers that are exploited for received signal strength (RSS) fingerprinting to less than $10 \%$ of the full set, the performances were still in a reasonable range.

S. Spinella et al. focus on the indoor environment as well and show an example of combining WLAN and RFID to aid one another. WLAN is employed to build a fingerprinting signal map to position the device. The RFID tags allow splitting the area into zones that support the fingerprinting algorithm to further gain in speed and accuracy. The theoretical results were evaluated and confirmed in a measurement campaign.

The second group of papers investigates systems that use GNSS as part of their evaluation. B. Motella et al. assess the impact of interference due to communications 
systems transmitting in close bandwidths to GNSS signals. The impact on GNSS is quantified by using interference error envelopes which measure the correlation of the distortion versus the characteristic of the interferer.

The next paper by C. Mensing et al. explores GNSS critical environments. The ubiquitous available cellular mobile radio systems are capitalized to improve acquisition and tracking of the position of the mobile phone. In addition, the mobility of the user is used to track the mobile phone and help to overcome the signal loss of GNSS in GNSS denied environments (e.g., urban canyons). The performance evaluation for the mobile radio differs between the different areas in a cellular radio system, for instance, proximity of the base station or cell edge.

The hybridization of multiple signal sources through nonlinear filters is studied by C. Fritsche and A. Klein. The authors apply their data-fusion algorithms to (raw) measurements in terms of time and received signal strength (RSS) from 2G mobile radio systems as well as to partial information (pseudoranges) coming from GNSS systems. The baseline assumption is that the radio base stations are all synchronized (based on GNSS timing information). They show that hybridization improves the localization method by increasing the accuracy of the global positioning system (GPS) in severe scenarios.

S. Sergi et al. discuss a ranging technique based on RSS for indoor scenarios by clustering mobile phones. The mobile phones perform ranging by using multiple measurement links (multiple base stations), and clustering is the enabling factor to attain reasonably accurate positioning.

Finally, $N$. Yi et al. investigate the concept of underlay cognitive radios (UCRs) which allows a secondary user to enter a primary user's spectrum. A location-aided UCR physical-layer model is established and analyzed. The positioning information is linked to the channel quality, and the knowledge of the terminal position is used to study new spectrum and power allocation techniques to optimize the capacity of the secondary user as well as the capacity penalty of the first user.

We would like to thank all authors and reviewers for their contributions. We do hope that this special issue may serve to promote further research in this new and exciting area. Finally, we would like to acknowledge the EU Research Projects WHERE (Contract no. 217033) and NEW$\mathrm{COM}++$ (Contract no. 216715) which aim at inspiring the development of new position-aware procedures to enhance the efficiency of communications networks and of new positioning algorithms based both on (outdoor or indoor) wireless communications and on satellite navigation systems.

Ronald Raulefs Simon Plass Marco Luise 

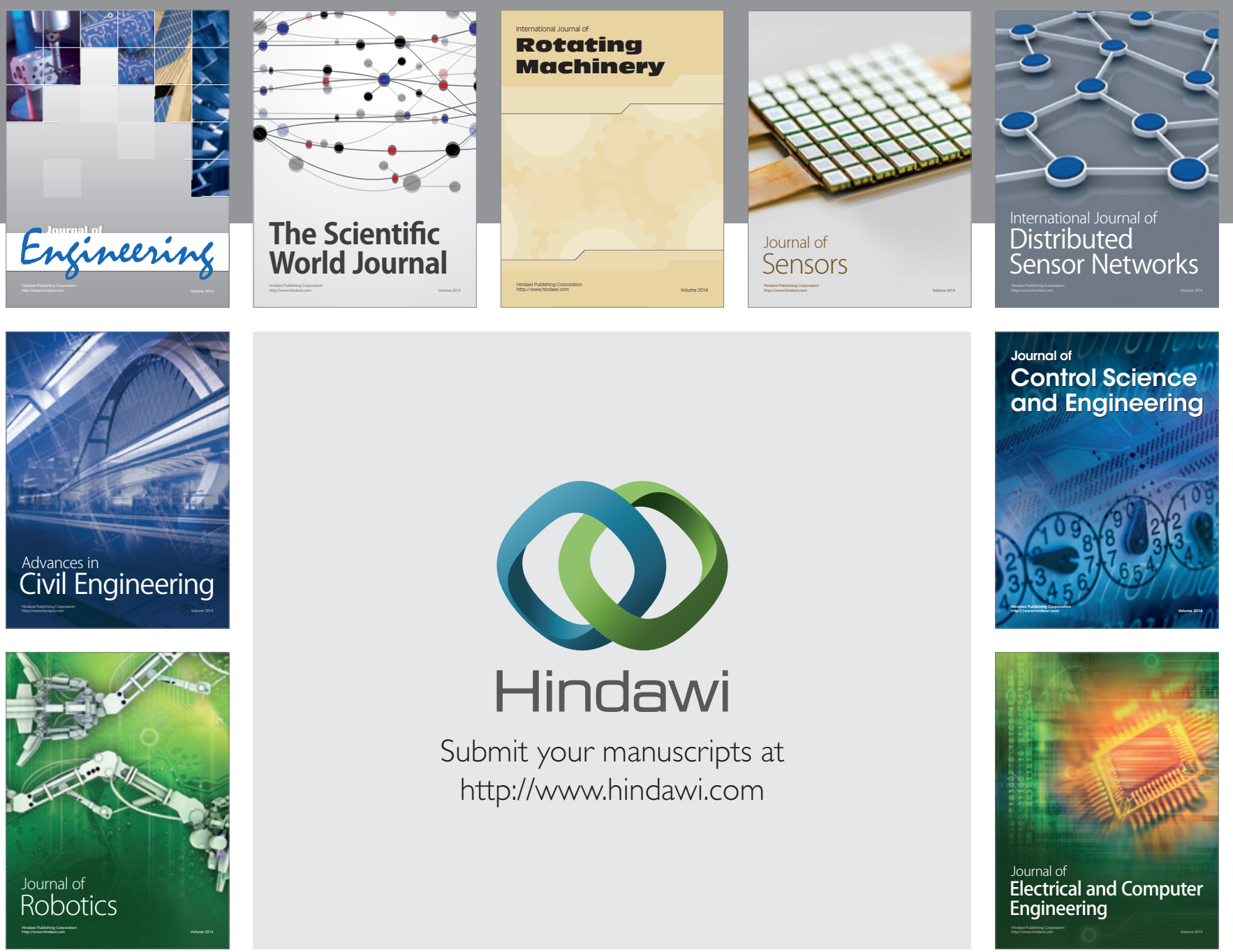

Submit your manuscripts at

http://www.hindawi.com
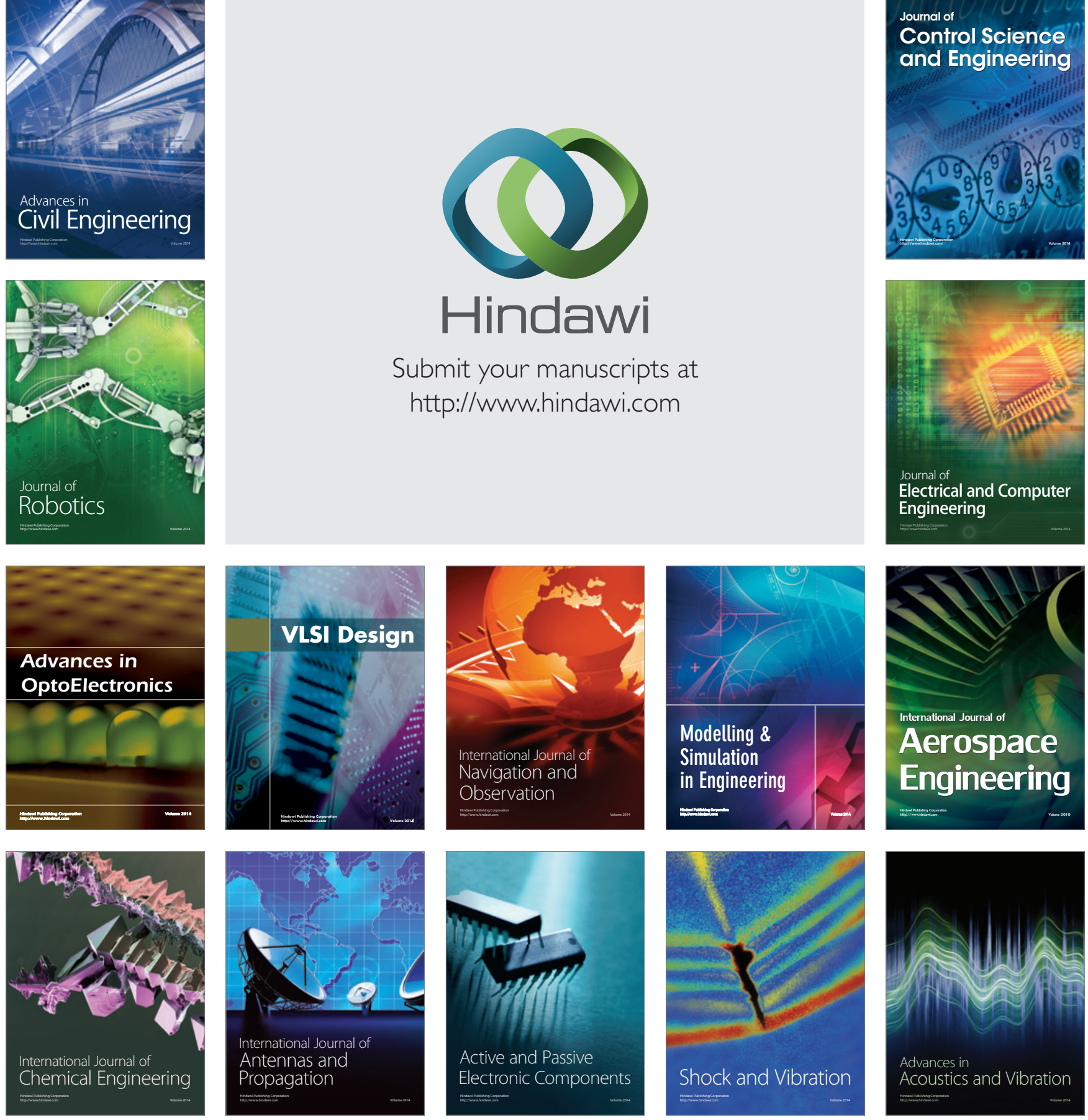